\title{
Der Radbaum - eine dendrologische Besonderheit im Palmengarten
}

\author{
Veit Martin Dörken \& ANnetTe HögGemeier
}

\section{Abstract}

The genus Trochodendron (Trochodendraceae) comprises a single species, Trochodendron aralioides SIEB. \& ZUCC. from east Asia. It is a medium sized evergreen tree. The species lacks vessels, the leaves exhibit idioblasts. Flowers are green, arranged in erected racemose inflorescences. The perianth is reduced, there are up to 70 stamens and about 11 carpels.

\section{Zusammenfassung}

Die Gattung Trochodendron (Trochodendraceae) umfasst nur eine Art, Trochodendron aralioides SIEB. \& ZuCC., die in OstAsien heimisch ist. Am Naturstandort wird dieser immergrüne Baum bis $20 \mathrm{~m}$ hoch. Die Art sowie die ganze Familie sind durch das Fehlen von Tracheen im Xylem und durch das Vorkommen von Idioblasten in den Blättern charakterisiert. Die grünlichen Blüten stehen in aufrechten Trauben. Das Perianth ist stark reduziert. Die Einzelblüten weisen bis zu 70 Staubblätter sowie rund 11 Fruchtblätter auf.

\section{Systematik}

Der immergrüne Radbaum (Trochodendron aralioides Sieb. \& ZUCC.) gehört zur Familie der Trochodendraceae. Diese Familie wird je nach Auffassung als monotypisch angesehen (HUTCHINSON 1979) oder umfasst außerdem die laubabwerfende Gattung Tetracentron (ENDRESS
1993, Mabberley 2008). Zu beiden Gattungen wird jeweils nur eine Art gestellt. Die Trochodendraceae werden vielfach als ursprüngliche $\mathrm{Fa}$ milie angesehen. Charakteristisch ist das Fehlen von Tracheen im Holz und die Ausbildung von Idioblasten in den Blättern (TAYLOR et al. 2009). Idioblasten sind Zellen, die sich in Größe, Bau

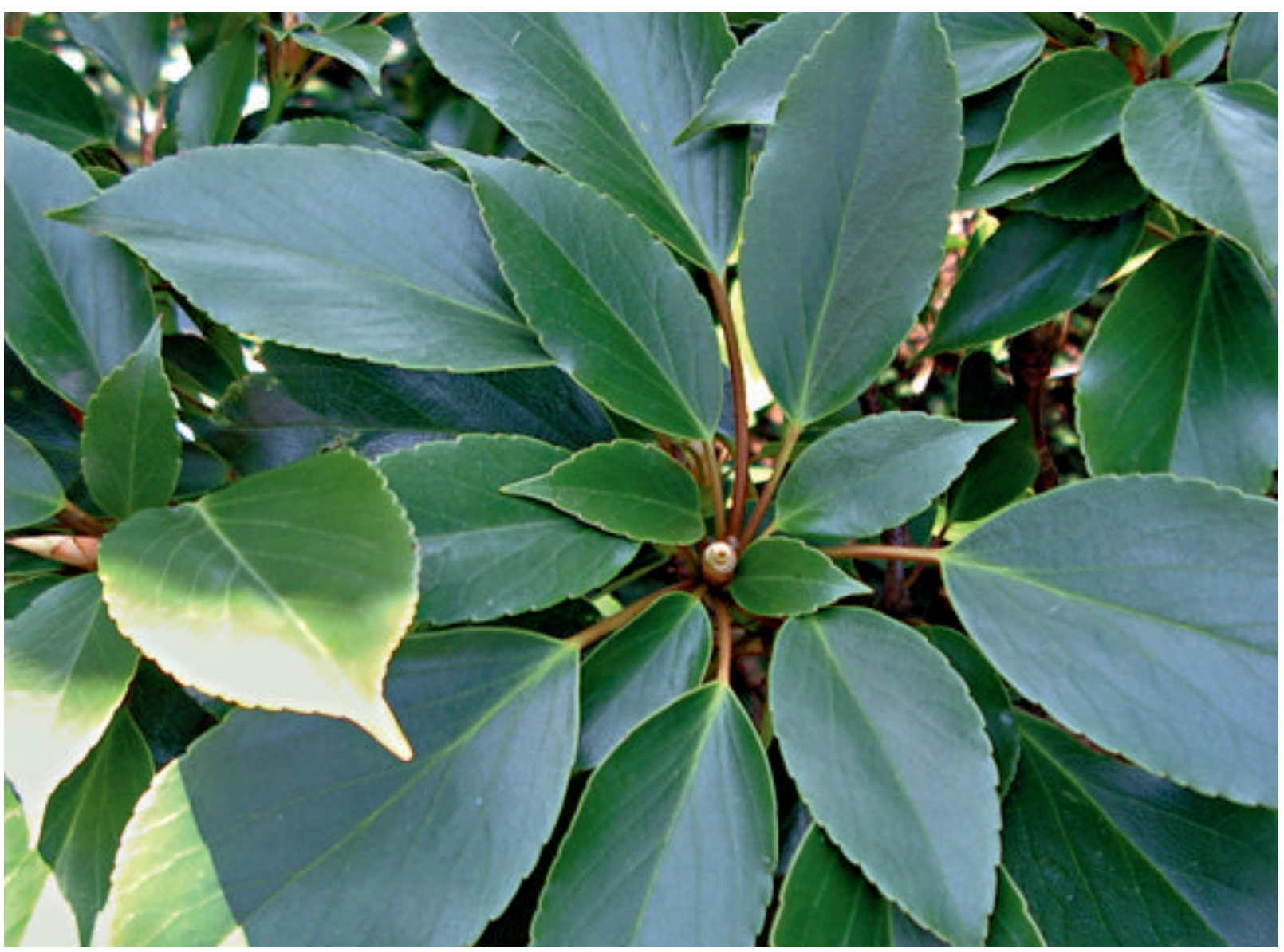


oder Inhalt wesentlich von ihrer Umgebung unterscheiden.

\section{Verbreitung}

Trochodendron ist fossil bereits aus Ablagerungen der späten Kreidezeit nachgewiesen und hatte früher ein wesentlich größeres Areal als heute (PIGG et al. 2001, 2007). In Kanada gibt es Funde von Trochodendron drachukii aus dem Eozän (TAYLOR et al. 2009). Heute ist der Radbaum nur noch in Korea, Japan und Taiwan heimisch, wobei er in Japan in Höhenlagen zwischen 300 und $2000 \mathrm{~m}$ und in Taiwan sogar bis $2700 \mathrm{~m}$ Höhe auftritt (ENDRESS 1993).

\section{3. Äußeres Erscheinungsbild und Bluiten}

Am Naturstandort erreicht das Gehölz immerhin Höhen um 20 m (MABBERLEY 2008). In mitteleuropäischen Gärten wächst der Radbaum an geschützten Standorten angeblich nur zu einem maximal $5 \mathrm{~m}$ hohen Großstrauch heran.

Die Rinde duftet beim Zerreiben aromatisch. Die derb-ledrigen, bis $15 \mathrm{~cm}$ langen, rhombischen bis eiförmigen Blätter stehen wechselständig und gehäuft an den Triebenden, Nebenblätter fehlen. Die Blätter haben einen drüsig gesägten Rand, eine glänzend dunkelgrüne Oberseite und deutlich hellere Unterseite. Von Mai bis Ende Juni erscheinen gelbliche bis grüne, $1-1,5 \mathrm{~cm}$ breite Blüten, die sich in bis $15 \mathrm{~cm}$ langen aufrechten, traubigen Blütenständen befinden. Kelch- und Kronblätter fehlen. Die Deutung der 2-5 kleinen Hochblätter an der Blütenachse als Rudimente einer ehemaligen Blütenhülle ist umstritten. Je Einzelblüte stehen 40 bis 70 Staubblätter vom Rand einer flachen, leuchtend frischgrünen Nektarscheibe radförmig ab, worauf der Name Radbaum (von gr. trochos = Rad) Bezug nimmt. Die 4-11 kreisförmig angeordneten Fruchtblätter sind mit der Blütenachse und im unteren Bereich seitlich unter-

Abb. 1 (S. 124): Zweig des Radbaumes.

Abb. 2 (oben): Blühender Radbaum.

Abb. 3 (unten): Fruchtender Zweig.

Abb. 4 (S. 126): Trochodendron-Blüte im Detail.
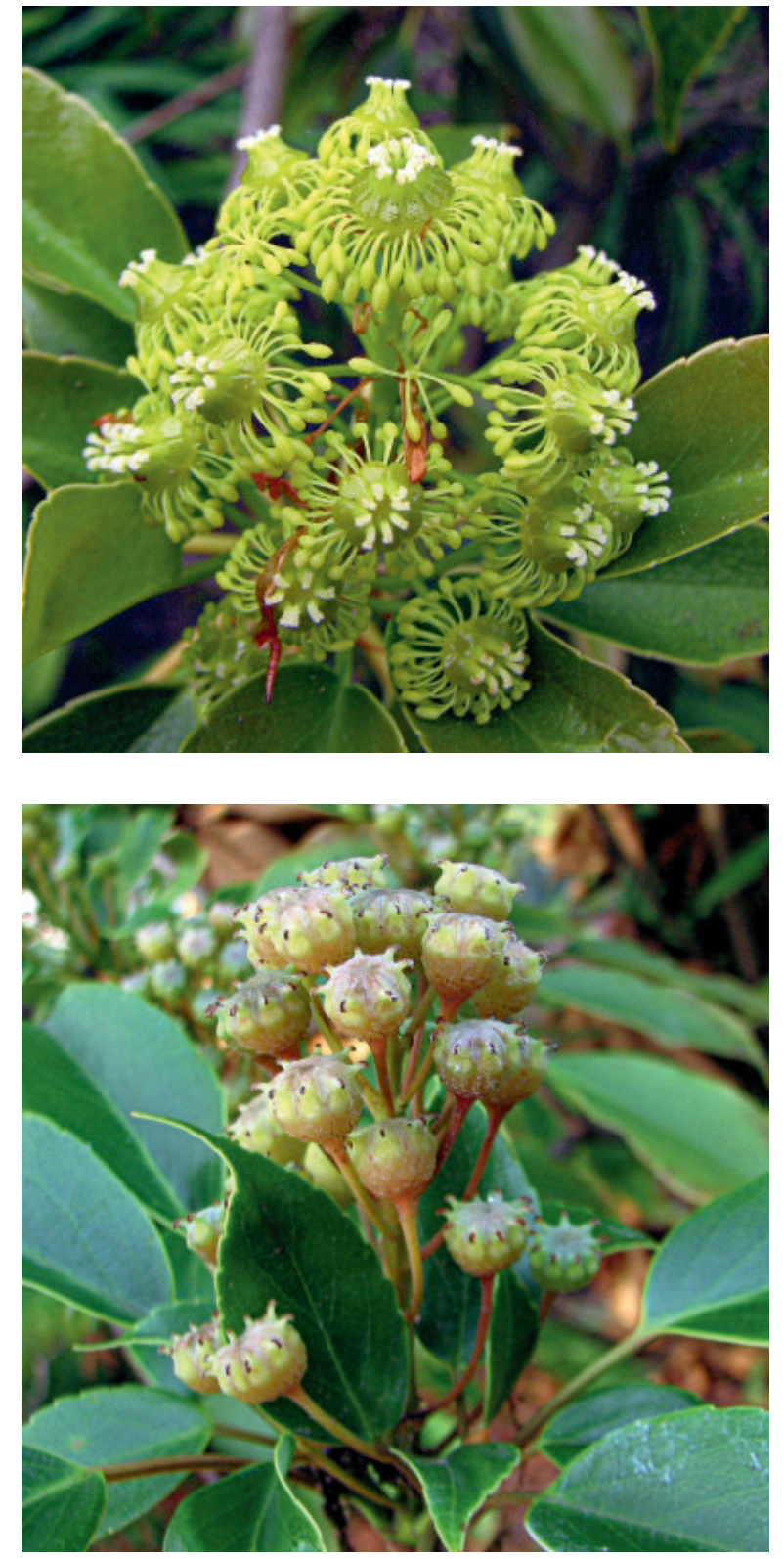

einander verwachsen, die freien Griffel sind deutlich nach außen gezogen. Die Blüten produzieren Nektar und werden von Insekten bestäubt (Huber 1991). Trochodendron bildet Sammelfrüchte, die sich aus kleinen, vielsamigen Balgfrüchtchen aufbauen (ENGLER 1964).

\section{Standort und Kultur}

Mit ca. 5 und 4,50 m Höhe zählen die beiden im Palmengarten an einem geschützten Standort vor dem Blütenhaus gepflanzten Individuen wahrscheinlich zu den größten Exemplaren in Deutschland. Für ein optimales Gedeihen benötigt der Radbaum voll sonnige bis licht schattige Standorte, die vor winterlicher Morgensonne 


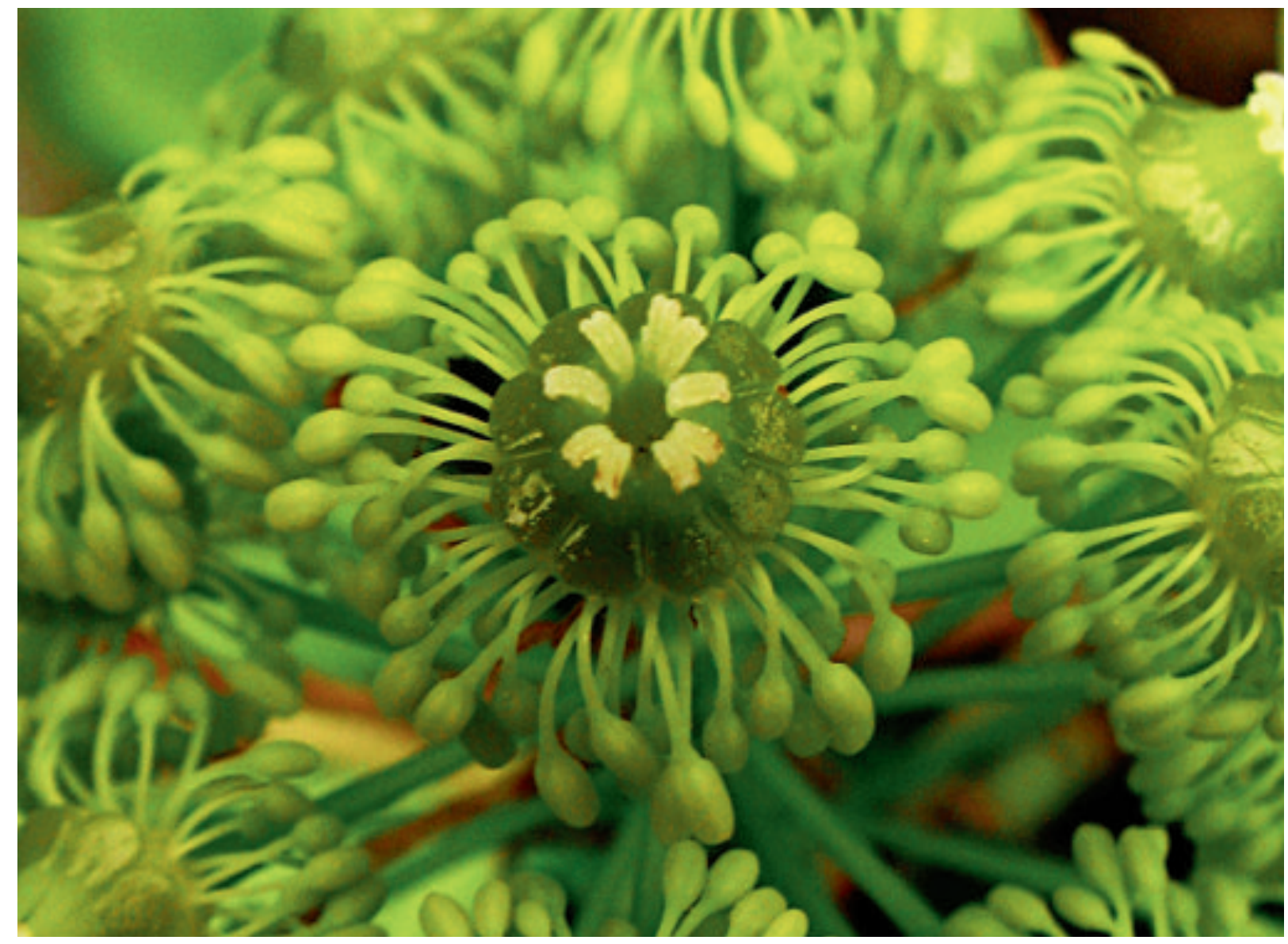

und austrocknenden Ostwinden geschützt sind. Der Boden sollte stets frisch bis feucht sein. Staunässe wird in der Regel nicht vertragen (EVERETT 1982), das Substrat sollte nährstoffreich sein. Empfindlich reagiert der Radbaum gegenüber flachgründigen Kalkböden (Kelly \& Hillier 2004). Trochodendron aralioides sollte daher auf schwach sauren bis neutralen Standorten gepflanzt werden, leicht alkalische Standorte werden auch noch toleriert. Der Radbaum ist ausreichend frosthart für die Winterhärtezone $7 \mathrm{~b}$, was Regionen entspricht, in denen die winterlichen Temperaturminima zwischen $-14,9^{\circ} \mathrm{C}$ und $-12,3{ }^{\circ} \mathrm{C}$ liegen. Trotz der beiden aufeinander folgenden Extremwinter 2008/2009 und 2009/2010 mit Temperaturminima um $-20{ }^{\circ} \mathrm{C}$ zeigten die im Palmengarten stehenden Exemplare nur geringe Frostschäden, der größere der beiden Bäume hatte im Februar 2010 einige deutlich braune Blätter.

\section{Literatur}

ENDRESS, P. K. 1993: Hamamelidaceae. In: KUBITZKI, K.: The families and genera of vascular plants. II, Flowering plants, Dicotyledons: Magnoliid, Hamamelid and Caryophyllid families. - Heidelberg.

Engler, A. 1964: Syllabus der Pflanzenfamilien,

12. Aufl. - Berlin.

EveretT, T. 1982: The New York Botanical Garden illustrated encyclopedia of horticulture. - New York. Kelly, J. \& Hillier, H. 2004: Bäume und Sträucher. 2. Aufl. - Braunschweig.

Huber, H. 1991: Angiospermen, Leitfaden durch die Ordnungen und Familien der Bedecktsamer. - Stuttgart. Hutchinson, J. 1979: The families of flowering plants, arranged according to a new system based on their probable phylogeny. - Königstein.

PigG, K. B., Wehr, W. C., \& ICKerT-Bond, S. M. 2001: Trochodendron and Nordenskioldia (Trochodendraceae) from the middle Eocene of Washington state, USA. Int. J. Plant Sci. 162: 1187-1198.

Pigg, K. B., Dillhoff, R. M., De Vore, M. L., \& Wehr, W. C. 2007: New diversity among the Trochodendraceae from the early/middle Eocene Okanogan highlands of British Columbia, Canada, and northeastern Washington State, United States. Int. J. Plant Sci. 168: 521-532.

TAYlor, T. , TAYlor, E. L. \& Krings, M. 2009: Paleobotany, the biology and evolution of fossil plants. - New York. 